\title{
Health-Related Quality of Life, Mental Health and Psychotherapeutic Considerations for Women Diagnosed with a Disorder of Sexual Development: Congenital Adrenal Hyperplasia
}

\author{
Matthew A. Malouf, Arpana G. Inman, Amanda G. Carr, Jill Franco, and Lindsey M. Brooks \\ Department of Counseling Psychology, Lehigh University, 111 Research Drive, Iacocca Hall, Bethlehem, Pennsylvania, PA 18015, USA \\ Correspondence should be addressed to Matthew A. Malouf, malouf@lehigh.edu
}

Received 29 January 2010; Accepted 25 March 2010

Academic Editor: Peter Allen Lee

Copyright (C) 2010 Matthew A. Malouf et al. This is an open access article distributed under the Creative Commons Attribution License, which permits unrestricted use, distribution, and reproduction in any medium, provided the original work is properly cited.

\begin{abstract}
Little is known about the long-term health-related quality of life (HRQL) and mental health outcomes for women diagnosed with congenital adrenal hyperplasia $(\mathrm{CAH})$, a disorder of sex development. Though recommendations for therapists exist, no research has empirically investigated women's experiences in therapy or their recommendations for therapy. Thus the purpose of the study was to investigate HRQL, mental health concerns of women with $\mathrm{CAH}$, and patients' recommendations for therapists. A qualitative methodology, consensual qualitative research, was used to answer these questions. Eight women with CAH participated in the study. Results from their interviews revealed six domains: physical functioning, psychological functioning, interpersonal processes and relationships, healthcare experiences of women with $\mathrm{CAH}$, recommendations for health care professionals, and systemic considerations. Domains were further broken down into categories with results yielding implications for both medical and mental health professionals and shedding light on issues related to normalcy, concerns both related and unrelated to $\mathrm{CAH}$, sex, and gender, and highlighting effective supports and therapeutic interventions.
\end{abstract}

\section{Introduction}

Conventionally referred to as intersex conditions and affecting an estimated 50,000 Americans [1], disorders of sex development (DSD) and best practices of care have remained a controversial topic in the health community. Affecting an estimated 1 in 15,000 births [2], congenital adrenal hyperplasia $(\mathrm{CAH})$ results from an inherited enzyme deficiency leading to an overproduction of adrenal hormones. In karyotpyic females (46, XX) this elevated level of androgenic hormones impacts development in utero by virilizing or masculinizing/defeminizing the genitalia [3]. Since CAH is often first treated by pediatric endocrinologists, surgeons, and urologists, research on outcomes and interventions has primarily centered on issues most clinically relative to these fields, specifically concerns related to sex of rearing, psychosexual outcomes thought to influence sex assignment such as gendered play in childhood, gender identity, and sexual orientation (see [4], for a review) and on ethical considerations related to treatment [5]. Thus, while CAH's impact on sexual and psychosexual development is wellestablished in the literature, its impact on health-related quality of life (HRQL), including mental health outcomes, is less clear.

In recent years, a multidisciplinary approach for the treatment of DSD has been recommended to also include gynecological, genetic, neonatal, psychological, ethical, and social work specialists working with children, adults, and their families [6]. Specific to mental health specialists, psychotherapeutic recommendations have been proposed [7], yet little is known about what interventions are most efficacious or what specific quality of life and mental health concerns are most salient for women diagnosed with CAH. Thus the aim of this study was to determine the manner 
in which having CAH impacts HRQL (defined as physical, functional, emotional, and social well-being), mental health experience, and counseling needs for women diagnosed with this condition.

\section{Health-Related Quality of Life Specific to Disorders of Sex Development and CAH}

Although the historical focus was primarily on the medical management of $\mathrm{CAH}$, within the past thirty years research has focused on understanding how chronic illnesses such as $\mathrm{CAH}$ impact quality of life (QoL) through measures specific to health-related quality of life (HRQL). HRQL typically focuses on those domains of QoL that would be directly affected by one's medical status including physical well-being (symptoms, impairments), functional wellbeing (daily living, role performance), emotional well-being, (coping, distress), and social well-being (relationship quality, family; [8]).

Findings on HRQL specific to women with CAH have been mixed but have generally revealed implications for identity and relationships. One study found that women with CAH had a significantly impaired HRQL compared to controls [9]. This study also revealed that women with $\mathrm{CAH}$ were presently engaged in fewer relationships (either heterosexual or homosexual) than controls. Although one must be cautious in implying that a lack of relationships inherently denotes impaired HRQL, women with CAH have often expressed concerns related to romantic partnerships, specifically fears of rejection, impacted body image, and selfesteem $[10,11]$. Though Kuhnle et al., [12] revealed no significant differences on a generic QoL measure, women with $\mathrm{CAH}$ did report trends of feeling less attractive, less pleasant in appearance, less erotic, less romantic, less feminine, and less confident compared to age-matched control women and were more often single, less sexually active and had less children compared to controls. Research also finds women with $\mathrm{CAH}$ have other issues relevant to their condition that may impact their quality of life, including those related to disclosing health-related information, weight and wellbeing, as well as having a chronic life-threatening condition [10].

Additionally, despite improving techniques, female identity may be compromised by imperfect appearing or performing genitalia, even after surgery. Indeed, CAH participants reported greater amounts of pain during intercourse $[11,13]$ and were only moderately satisfied with the cosmetic appearance of their genitalia, rating their appearance lower than physicians' ratings [14]. Liao [15] reports that women diagnosed with a DSD such as CAH may also have difficulty disclosing about their atypical genitalia to potential sexual partners. Shame, secrecy and stigma often accompany ambiguous genitalia and the surgeries are, at times, framed as "normalizing" [10]. An emphasis on normalcy [16] versus a focus on the enjoyment of sex and intimacy may in turn lead to anxiety and/or avoidance of sexual intimacy [15]. This potentially explains the low rates of romantic partnerships and sexual activity reported in the investigations of women diagnosed with CAH $[9,11,12,17,18]$. Furthermore, for women who may want to get pregnant, problems with fertility (see [19] for a review of fertility research) may also impact willingness to pursue relationships, and some individuals have reported the inability to satisfy a potential mate's needs for children as a barrier to marriage (C. Migeon, personal communication, January 8, 2006).

\section{Mental Health Concerns and CAH}

$\mathrm{CAH}$, a chronic illness that may require repeated surgeries and challenge notions of identity and normalcy, influences those aspects of HRQL related to emotional wellbeing. Nonetheless, this relationship has only recently been explored in the literature. The sparse research on mental health outcomes related to disorders of sex developments in general has focused primarily on the presence of psychopathology. Some evidence suggests that children with a disorder of sex development are at risk for psychological concerns, with roughly $40 \%$ of participants presenting with at least one DSM-IV diagnosis and an additional 20\% presenting with less severe problems related to learning of their diagnosis and/or surgical interventions [20]. Yet, findings are mixed with another study reporting no differences between adult participants diagnosed with a DSD and both healthy and chronic illness control groups on measures of general mental health, posttraumatic stress, and depression [11]. The DSD group, however, did exhibit higher trait anxiety and lower self-esteem than unaffected controls, but were no different than those with a chronic congenital disorder unrelated to sex development.

Similarly, findings for females diagnosed with $\mathrm{CAH}$ are varied. Although some participants in one study met diagnostic criteria for DSM diagnoses similar to those reported in the previous study (i.e., major depressive disorder, social phobia, panic disorder, generalized anxiety disorder), their overall scores on measures of depression, social adjustment, and eating behaviors and attitudes were within normal ranges [21]. In contrast, Johannsen et al. [9], found greater mental distress in this population as well as greater suicidal ideation and attempts when compared to controls. Similarly, when compared to participants with other DSD diagnoses, women with $\mathrm{CAH}$ had more suicide attempts. Women with $\mathrm{CAH}$ also had significantly higher scores on an anxiety measure, whereas participants with different DSDs did not differ from controls. This suggests that women with $\mathrm{CAH}$, in particular, may be at a greater risk for psychopathology. Though research has begun to explore factors associated with poorer mental health (e.g., self-concept, personality traits, interpersonal functioning [7]) for women with $\mathrm{CAH}$, no study has directly examined the influence of CAH on HRQL and mental health.

\section{Research on Psychotherapeutic Interventions}

Though the above findings are useful in developing recommendations, little is known about what empirically-based psychotherapeutic interventions are most useful for this population. Based on its success in reproductive medicine, group work has been recommended for individuals with 
a DSD [15]. Individual therapy focusing on interpersonal relationships has also been suggested [6], yet stigma exists surrounding seeking psychological help, especially for those diagnosed with a DSD [10]. It is thought that support groups may be beneficial in ending stigma and isolation [6], and some women with CAH do take advantage of them [17]. Support for families is an additional recommendation, as some women with a DSD report difficulty talking about condition-related issues with family members [15]. This family intervention may be especially beneficial given findings that psychopathology in individuals with a DSD occurred twice as often among those with children and parents who did not receive counseling at diagnosis [20].

Given the inconsistent findings for HRQL and mental health concerns as well as limited research regarding psychotherapeutic interventions for women with $\mathrm{CAH}$, a qualitative methodology, consensual qualitative research (CQR) $[22,23]$, was deemed a valuable approach to exploring these important treatment issues. Given the history of treatment for DSD and ethical concerns regarding treatment decisionmaking, CQR was also determined to be a culturally sensitive method in that it gives much-needed voices to individuals. Similarly, it does not seek to present one universal understanding and instead provides diverse perspectives based on the participants' experiences; an important consideration given differences within the population. Other authors have also reported that interviews were more successful in helping participants disclose thoughts and feelings regarding their condition [24] than paper and pencil measures. CQR was also chosen specifically for its demonstrated usefulness in gathering a large amount of data from a small sample, as research on this population is often constrained by small numbers given the rarity of this condition.

\section{Materials and Methods}

\subsection{Measures}

5.1.1. Demographic Questionnaire. This included characteristics like race, age, and so forth, as well as CAH diagnosis, medical, surgical, and psychiatric histories.

5.1.2. SemiStructured Interview Guide. Unpublished data from a longitudinal study [14] as well as consultations with various stakeholders informed the development of appropriate and sensitive questions. HRQL questions included inquiries about physical, functional, social, and mental/emotional well-being. Given findings on social outcomes and existing recommendations [7], questions assessing support group use were included. Similarly, as gaining psychotherapeutic knowledge was a goal of the investigation, questions assessing therapy/counseling use, experiences, and recommendations were included. Openended questions were employed to promote full explanations with prompts/probes used for clarification.

5.2. Participants. Eight participants were included, all identified as females. All identified as Caucasian or White and ages ranged from 23 to $51(M=33$, median $=31)$. Seven were diagnosed with the salt losing form of $\mathrm{CAH}$, and one with simple virilizing type. Six participants identified as heterosexual or straight with the two identifying as lesbian or queer. All had at least an undergraduate degree with the average and median years of education being 18. All had at least one genital surgery (clitoroplasty and/or vaginoplasty, range $=1$ to 3 surgeries, median $=2$ ), with a mean age of 3.88 years at first surgery $($ range $=6$ to 18 , median $=1$ ). All participants reported taking medication for CAH. Regarding surgery/treatment, participants were treated at different facilities representing diverse geographic locations in the US. Additionally, all but one participant reported a current or past mental health concern, six sought counseling for it and three sought counseling for issues surrounding CAH.

5.3. Procedure. Announcements were placed in DSD/Intersex/CAH support groups including the Congenital Adrenal Research Education and Support Foundation (CARES) and the Congenital Adrenal Hyperplasia Support Forum, through the NIH's Clinicaltrials.gov website and a standalone study website. Multiple sources were used due to concerns about how unique clinical experiences might steer individual towards or away from various medical, research, or support organizations. Upon contacting the researcher, a letter was mailed explaining the study and inclusion criteria along with an IRB-approved consent form. The demographic questionnaire was mailed as a follow-up. After returning the questionnaire, participants were screened for inclusion on the basis of self-reported diagnosis (classical $\mathrm{CAH}$ ), biological sex (female), and age (21-50). Geographic location, treating-hospital/s, surgical history, severity (saltlosing or simple virilizing), and referral method (support group versus other) were monitored in attempts to avoid over-representation. Participants were then contacted by the primary investigator to set up a phone interview, lasting approximately one hour.

5.4. Research Team. As recommended by Hill et al. [23], prior to conducting the analysis, the team shared their individual identity labels as well as general biases about the topic. The team consisted of three counseling psychology doctoral students; one identified as male, aged 30, and two identified as female, aged 29 and 27. The auditor was also a counseling doctoral student and identified as female, aged 27. Two members identified as queer, one as queer/bisexual, and another as heterosexual. Additionally, none were diagnosed with CAH or any DSD. All were trained in $\mathrm{CQR}$ and had previously been engaged in multicultural counseling research that included its use. One had experience working with individuals diagnosed with $\mathrm{CAH}$, two noted their pre-existing awareness of intersexuality but hoped to increase their knowledge, whereas one reported only having been exposed to the topic via a television program and interactions with others on the team. Common biases included believing that participants' $\mathrm{CAH}$-related identities were different than dichotomous sexuality or sex/gender identities, that individuals may report shame surrounding 
their diagnoses/treatments, that family considerations would be important, and that this population was, at least theoretically, similar to other historically marginalized populations in regards to mental health.

5.5. Analysis. Analysis followed methods from Hill et al., [23] and consisted of the following: transcription, domains, core ideas, and cross-analysis. Each step was checked either by the participants or an auditor with feedback then incorporated by the team prior to continuing. First, interview transcripts were mailed to participants for verification. Three participants responded that the transcripts accurately reflected their interview, and five did not respond. All transcripts (cases) were then reviewed by the team and general thematic domains were developed. After coding individually, team members then met for consensus on how to split each case into domains. Following auditing, each case was summarized/paraphrased (by domain) into core ideas, with team members taking turns leading by sharing their cores for an entire case. The final step consisted of a cross-case-analysis during which the team derived categories and subcategories from all participants' data within single domains. The team then came to consensus about exact placement of core ideas under each category/sub category and sent these results for auditing. Lastly, frequencies for each category were calculated based upon the number of cases that had core ideas within a category.

\section{Results}

Six domains described the participants' experiences and sub jective views of how having CAH impacted their HRQL, their mental health, and their recommendations for counseling (Table 1). Within each domain categories revealed general, typical, and variant themes, which, for some categories were further clarified by sub categories. Hill et al.'s [23] standards were used to determine frequency cutoffs with general representing the majority (7-8) of cases, typical representing more than half (6) of the cases, and variant for less than half (2-5) but more than one case.

6.1. Physical Functioning. This domain revealed seven categories encompassing issues related to the impact of illness and treatment on physical/physiological functioning. Generally, participants reported feeling like they were functioning normally, a category comprised of two subcategories. The first category, able to function well with no or minimal $\mathrm{CAH}$ related restrictions, was typical and included statements like, "I function day to day (and) go to work like everybody else." The second, a variant subcategory, was normal functioning except for having to take medication daily.

Participants generally commented on the impact of $\mathrm{CAH}$ on sexual functioning, ranging from difficulty getting pleasure/orgasm from sex to feeling that they could have sex just like anyone else to stating that penetrative sex was not an issue given the gender of their romantic partners. Participants also generally noted an increased risk for and sensitivity to sickness, including both a history and fear of being sick or tired (e.g., "I was at a friend's house. I did not want to have them take me to the hospital. During (the) couple of days that I was sick it was on my mind the whole time").

Under the general category medication, four subcategories emerged, all of which were typical. First were observations regarding medical regimens, for example, having to take medication daily or wear a medic alert bracelet and information regarding the level of their doses. Second were observations about stress dosing with participants sharing their practice or importance of altering their medications based upon stress. Third were general side effects from medication, including problems with blood pressure, weakened teeth/bones, and sleep/energy problems. One participant noted that sensitivity to light made it hard for her to do things outdoors. Lastly, a subcategory titled weight concerns from medication included reports of weight gain, difficulty losing weight, and a resulting ambivalence about medication.

Finally, within this domain there were two additional categories that were typical in nature. The first, physical side effects of having $\mathrm{CAH}$, mainly included observations about hirsutism and short stature. Second were comments related to adrenal crises and hospitalizations, both in childhood and adulthood (e.g., "I had a bad crisis 4 years ago. I did not show up for work, and when someone called and I did not make sense they sent an ambulance. The paramedics thought I was dead cause I was unresponsive"). Lastly, in the variant reproductive functioning three participants noted issues with their fertility surrounding CAH, while one stated, "I will not be able to have kids and I do not think it's related to CAH."

6.2. Psychological Functioning. This domain encompassed seven unique categories, three of which were general and all of which described various processes related to emotions and the mind. The first general category, processes involved in understanding or becoming aware of $C A H$, described moments of awareness and acceptance and included desires to understand more. For example, upon reading details about her condition, one participant stated, "In the letter it talked about having ambiguous genitals, and I was like, just devastated when I read that." Other participants reported positives including learning to accept their condition and an ability to relate to others or increased self-awareness, as one participant reported, "I think this thing has resulted in me just being more aware or in tune in a good way."

The next general category was understanding of identity and included two opposing subcategories, both of which were variant. On one hand, participants talked about feeling normal, for example, "Some people see CAH as an identifier. No, my identity is me, I have a certain job, I have a masters degree." Yet, participants also spoke about feeling different with statements such as, "I have always had this sense of not quite fitting in and I wonder now if (CAH) contributed."

Another general category was emotions and cognitions related to $C A H$, including two subcategories, negative, which was general, and positive, which was variant. Negative included things like general irritability, stress, problems with confidence, and reactions to treatment such as frustration, anger (e.g., "I felt violated at the hospital after being vaginally 
TABLE 1: Representation of cases within each category and subcategory across each domain.

\begin{tabular}{|c|c|}
\hline Domain/Category/Subcategory & Frequency* \\
\hline Domain 1: Physical functioning & general \\
\hline Normal functioning & general \\
\hline (i) Able to function well with no or minimal CAH-related restrictions & typical \\
\hline (ii) Normal functioning except for having to take medication daily & variant \\
\hline Sexual functioning & general \\
\hline Increased risk for sickness & general \\
\hline Medication & general \\
\hline (i) General medical regimens & typical \\
\hline (ii) Stress dosing & typical \\
\hline (iii) General side effects from medication & typical \\
\hline (iv) Weight concerns from medication & typical \\
\hline Physical side effects of CAH & typical \\
\hline (i) Adrenal Crises and hospitalizations & typical \\
\hline (ii) Reproductive functioning & variant \\
\hline Domain 2: Psychological functioning & general \\
\hline Processes involved in understanding or becoming aware of CAH & general \\
\hline Understanding of identity & general \\
\hline (i) Normal & variant \\
\hline (ii) Different & variant \\
\hline Emotions and cognitions related to $\mathrm{CAH}$ & general \\
\hline (i) Negative & general \\
\hline (ii) Positive & variant \\
\hline Mental health concerns & typical \\
\hline Sexuality & variant \\
\hline (i) Gender identity and role & variant \\
\hline (ii) Concentration and learning issues & variant \\
\hline Domain 3: Interpersonal relationships and processes & general \\
\hline Disclosure and others' awareness and knowledge of CAH & general \\
\hline (i) Impact of CAH on others & general \\
\hline (ii) Interaction of CAH's genetic component with relationships & general \\
\hline (iii) Utilization of support groups & general \\
\hline Supportive people and behaviors & general \\
\hline (i) Parents & general \\
\hline (ii) Other important relationships & general \\
\hline (iii) Romantic Partners & typical \\
\hline Unsupportive people and behaviors & typical \\
\hline Impact of $\mathrm{CAH}$ on dating & typical \\
\hline Having never met anyone else with CAH & typical \\
\hline Domain 4: Healthcare experiences of women with CAH & General \\
\hline Physicians & general \\
\hline (i) Physician's knowledge of CAH & general \\
\hline (ii) Negative medical interventions & general \\
\hline (iii) Positive medical interventions & typical \\
\hline (iv) Referrals & typical \\
\hline Mental health clinicians: therapists, psychologists and psychiatrists & general \\
\hline (i) Reasons for and goals of mental health treatment/therapy & general \\
\hline (ii) Positive psychotherapeutic interventions & general \\
\hline (iii) Negative psychotherapeutic interventions & variant \\
\hline (iv) Clinicians' knowledge of $\mathrm{CAH}$ & variant \\
\hline Intrapersonal processes in healthcare treatment & general \\
\hline
\end{tabular}


Table 1: Continued.

\begin{tabular}{lc}
\hline Domain/Category/Subcategory & Frequency* $^{*}$ \\
\hline Domain 5: Recommendations for healthcare professionals & general \\
general \\
typical \\
The need for more education about CAH & typical \\
(i) Things to consider when treating individuals with CAH & typical \\
(ii) Emotional impact & typical \\
Medical considerations & variant \\
Connect individuals with support groups & variant \\
The importance of access to therapeutic services & general \\
\hline Domain 6: Systemic considerations & typical \\
Generational and cohort differences in women with CAH & typical \\
Availability and appropriateness of care & typical \\
Insurance and other financial concerns related to CAH & variant \\
Societal biases and expectations &
\end{tabular}

$*$ general $=7-8$ cases, typical $=6$ cases, variant $=2-5$ cases.

dilated. My mom was told that I was acting like someone who was sexually abused. I believe I was traumatized by the procedure; it haunts me to this day"). Positive included statements such as, "I never had any sense of shame or anything associated with it (genital surgery)."

Typical for this domain was the category mental health concerns, and this included participant and family mental health and substance abuse histories. According to participants, some concerns were directly related to CAH, (e.g., "(after having a second surgery) I actually did get depressed and (was) diagnosed with depression the following summer. (Thinking back-the psychologists and psychiatrists ask what started it. I think that kinda started it; I felt like I could not talk to anyone and it was not that I wanted to talk a lot about it"), while others were unrelated (e.g., "I do not think my bipolar was caused by the CAH, because others in my family are bipolar and schizoaffective").

The remaining data from this domain fell under three variant categories. Sexuality included statements about understanding both attraction (either to men, women, or both) as well as identity (e.g., "I found out that I'm gay and I'm very happy with that"). Gender Identity and Role was comprised of statements including, "I sweat a lot and I just feel like a man sometimes," and "growing up I got the message it was ok to be a tomboy but that I needed to know how to be a lady too. I was taught how to cook, clean, sew, etc." Lastly, some participants reported concentration and learning issues, such as, "Growing up I was hyper or I was tired and it was just hard and focusing, sometimes I get ... hyper focused or I'd just totally screw around. That still happens now."

6.3. Interpersonal Processes and Relationships. This domain included eight categories pertaining to interpersonal or relational issues as a function of having CAH. Generally, disclosure and others' awareness and knowledge of $\mathrm{CAH}$ described processes related to telling others about CAH as well as their understanding and reactions. Some selected whom they told (e.g., "I am socially assumed to be a man a lot. Now, I do not correct people when they say "yes, sir" unless it is going to be a continuing relationship"), and some selected how much they told (e.g., "If I travel with someone I want them to know I carry a stress dose but that's about it"). Some participants seemed unsure of who actually knew or how much was known about their CAH. For example, one participant shared, "My family on my dad's side... I'm sure they knew or that they know but they do not know the extent of what's wrong with me and it's untalked about because they're weird in the first place and ... I think its taboo for them. On my mom's side of the family, they are more open."

Also general was the impact of CAH on others. This category included; negative feelings and experiences by family members and friends like frustration, guilt, and embarrassment as typified by the following quote: "He (husband) does not like it because it's frustrating because I'll go months and months being sick and there's no light at the end of the tunnel," as well as no impact on friends and family. The interaction of CAH's genetic component with relationships was also general and included past issues regarding heredity, for example, "There are only two times I have seen my parents feel guilty about passing on CAH," as well as future considerations such as, "I've thought about it and thought about if I would be able to pass it along to my children."

Utilization of support groups was a general category and captured participants' experience in or desire to be involved in various organizations or support groups. Some participants spoke about organizations like Congenital Adrenal Hyperplasia Research and Support Foundation (CARES) or congenitaladrenalhyperplasia.org with statements including, "I think the best thing I found was this website where we all share and now that makes me feel less alone." Additionally, one participant was uninterested in support groups saying, "My endocrinologist has suggested that I go to support groups but (I) never really thought about or felt the need."

Supportive people and behaviors were also identified generally within participants' data. Three subcategories were present with parents and other important relationships being general and romantic relationships being typical for 
participants. Supportive parental behaviors included helping with medical management (e.g., "Because my mom was a nurse she was able to ask questions and keep on top of my medications in a way I'm not sure other people would have") and normalizing the condition (e.g., "I really am grateful to my parents for never making me feel odd or sick or anything or ashamed"). Other important relationships included siblings (e.g., "My brother's very protective of me"), friends (e.g., "I would have to say if I had to be at any friend's house, that would have been the best place to be just because they are more mature and they would have taken care of me if I needed it"), religion (e.g., "Going to church again has helped me accept CAH"), and doctors (e.g., "My doctor, my mom, and two support groups are my only support"). Romantic relationships were exemplified with comments such as, "My romantic relationship is very good."

Unsupportive people and behaviors were also typically present in participant interviews. Included in this category were parents and siblings, reflected in the following statement: "It really hurt me to know my siblings resented me because I did not get abused though I know where they're coming from"). Also included were coworkers, as one participant stated, "I was working with a psychiatrist who asked me point blank, "was I trying to have a sex change, you know, was I pre-op?"” with the facial hair and stuff like that, and I had a really good relationship with the guy but it still just pissed me off that he said it," and people in general, for example, "I want people to understand where I'm coming from, but I feel frustrated because people tell me I do not look like I have a problem."

Also typical was the impact of $\mathrm{CAH}$ on dating. Within this category some participants reported a negative influence (e.g., "In my 20s and high school it was really hard; everybody else was dating and saying how fun it was and all of that and I was even afraid to go out with anybody"), whereas others reported no impact on dating. Lastly, and also typical, participants reported having never met anyone else with $\mathrm{CAH}$.

6.4. Healthcare Experiences of Women with CAH. This domain described women's experience with health care (including both physical and mental health) and was comprised of three categories, all of which were general. The first focused on experiences with physicians and contained several sub categories. Comments regarding physicians' knowledge of CAH were general and included statements about family doctors (e.g., "I was just lucky that my doctor knew the condition so that he could tell that there were issues with me"), endocrinologists (e.g., "I do not even feel that most endocrinologists I go to know much about the condition"), and other physicians (e.g., "I had to educate the (athletic) team's doctor at school because he just freaked out about the whole idea of (me) having adrenal insufficiency and the chance of getting hurt"). Also within this category were negative medical interventions, and participants often described physicians overmedicating patients or not listening and ignoring. This was exemplified by one participant's statement, "At the time my doctor told me (the weight gain) was not from the medication, that I was just pretty much lazy and was eating all the time and not exercising and so, when they finally put me on a different medication, which is prednisone, from there I lost all the weight." Positive interventions were typical and included comments such as, "This doctor I have down here listens ... and he understands and he actually asked me if he could be my doctor when we sat down and talked when I moved down here." Also typical were physicians' abilities to make appropriate referrals (e.g., to other physicians, research studies, or support groups).

The next general category focused on experiences with mental health clinicians including therapists, psychologists and psychiatrists and revealed four subcategories. First, participants generally described reasons for and goals of mental health treatment and/or therapy. Three participants were reportedly forced into treatment after suicide attempts or overdoses, and participants sought treatment voluntarily for issues both related to CAH (e.g., "The third time I got counseling was for eating issues and $\mathrm{CAH}$ came up in that when we got deeper into them") and unrelated to CAH (e.g., "I just got overwhelmed with work and school and everything else, so I found a licensed therapist"). A second general sub category described positive interventions, including those related to CAH like accepting and exploring $\mathrm{CAH}$ and its relation to other issues or simply listening and empathizing (e.g., "It felt good to talk to someone. You know, all the years I always held it in and did not talk to anybody about my feelings about the health problem ... I felt more content after I started sharing my feelings"), as well as those interventions unrelated to CAH (e.g., relaxation techniques for anxiety). Negative interventions were variant and primarily included comments about bias and lack of understanding, (e.g., "She was very opinionated and very judgmental, if I remember correctly. I felt like, "I do not need to pay you to tell me to judge me." Her job was to help me work through whatever issues I'm dealing with"). Lastly, and also variant, was the sub category clinicians' knowledge of $C A H$, which spoke to mental health workers' prior knowledge or lack of knowledge about CAH.

The last general category, intrapersonal processes in healthcare treatment, encompassed participants' internal thoughts, feelings, and reactions within the context of treatment. Examples included, "The only time I really think about it (CAH) is when I go to the doctor" and "It feels funny trying to explain to a shrink, "yeah, they built me a vagina and they cut off my nerve center."'

6.5. Recommendations for Healthcare Professionals. This domain included five categories of recommendations for medical and mental health professionals. The first and only general category was helpful therapeutic traits and was specific to mental health clinicians. This captured helpful traits of therapists (e.g., "A sickly therapist who could understand that sickness"), therapeutic approaches and techniques (e.g., "You still kind of have to fall back to what the individual needs of that person are"), and foci of therapy (e.g., "Counseling exploring family dynamics and relationships would be helpful").

Two categories were typical and applied to both medical and mental health professionals. The first, the need for more education about $\mathrm{CAH}$, emphasized the importance of 
increasing basic awareness and knowledge about the disease. The second, things to consider when treating individuals with $C A H$, went into greater detail about specific things for professionals to be aware of and contained two subcategories. The first emphasized the emotional impact of having $\mathrm{CAH}$ (e.g., "Doctors should not dismiss emotional problems that might be connected to CAH"). The second included comments for therapists about the medical considerations related to CAH (e.g., "Appropriate (psychotropic) medication and when to consult an endocrinologist").

Finally, two variant categories emphasized the need to connect individuals with support groups (e.g., "It'd also be good for them to have resources for you to go to. If they were able to find a good (internet message) board, like if you did not know about CongenitalAdrenalHyperplasia.org, they could inform you about it and you could talk to other people,") and the importance of access to and timing of therapeutic services for both individuals (e.g., "Counseling around the time of surgery would be helpful,") and families (e.g., "You have two healthy parents, and then suddenly they have got a sick baby that's dependent on drug therapy and mean doctors and stuff, but the kid just has it; the parents have to deal with it").

6.6. Systemic Challenges. The last domain spoke to systemic challenges faced by our participants and contained four categories. The first typical category was generational and cohort differences in women with $C A H$ and contained participant observations about differences between individuals with $\mathrm{CAH}$ of different ages as well as over time, as knowledge about CAH increases. For example, some participants noted the lack of interest in, or resources for, adults compared to those for children, while several observed changes in how doctors interact with patients. One participant stated, "I do know that there was not as much information given at that time ... you know, as far as stress dosing. It might be that we did not even know about it back then, that we as the patients did not know about it. I do not know what the medical community was doing then but there was a lot less, you know, interactions between doctors and patients at that time."

The next two categories, availability and appropriateness of care and insurance and other financial concerns related to $\mathrm{CAH}$, were both typical, and related to concerns about receiving adequate health care. The first included comments about difficulty finding experts, especially for adult patients/clients. The second included statements like, "We do not make much money so just thinking about how to financially be able to afford counseling is one thing," and "I feel so unsafe out here because I do not have any type of insurance." The last category in this domain was variant and spoke to societal biases and expectations, including statements such as, "In our society if you're a little different people look at you weird".

\section{Discussion}

The aim of this study was to determine the manner in which having $\mathrm{CAH}$ impacts HRQL, mental health experiences, and counseling needs for women diagnosed with this condition. Though findings suggest that $\mathrm{CAH}$ does indeed influence aspects of all of the above, the results both confirm and extend previous findings. Specifically, despite some common concerns related to their condition, our participants understood themselves as functioning normally. Most importantly, they indicated that for women with $\mathrm{CAH}$, feelings of normalcy are not solely explained by physical or functional outcomes but that the social and emotional well-being aspects of HRQL play an important role in recommendations for treatment. These findings provide critical information for both medical and mental health practitioners working with women diagnosed with CAH.

Our findings support previous results indicating that aspects of HRQL are indeed influenced by CAH [15]. In fact, participants shared concerns across all domains of HRQL. However, according to the results of the current study, certain aspects of HRQL, especially some physical concerns, may actually be more salient of a concern for clinicians than for individuals. For example, although the desire to maximize fertility is something which often plays an important role in determining the sex of a newborn with DSD, and was a concern reported by women with $\mathrm{CAH}$ in another study [7], this theme was variant, with just half of our participants bringing up fertility and only half of those mentioning it as a physical problem. Similarly, though research has often focused on concerns with sexual/genital functioning (see [14] for review), for many women in this study it was a non issue given their satisfaction with their sexual functioning or lack of interest in penetrative sex. Surprisingly, many concerns which were salient to participants did not abnormally impact their functioning as they saw it (e.g., having to take medication), and women described themselves as functioning normally both in childhood and adulthood. They attributed this to parents, friends, and partners not treating them as sick, to effective medical interventions, and to their own coming to terms with CAH.

The "processes involved in understanding or becoming aware of CAH" was a blend of both positive and negative experiences. The former were related to acceptance and support. The latter were most often associated with discovering specifics about their history or the extremes of CAH (sometimes unintentionally as one participant did when doing research for a school project) and were experiences that could have been circumvented through increased education and openness regarding the condition. In relation to social functioning, results from the study provided additional support to previous findings, which suggest that $\mathrm{CAH}$ does negatively impact participants' relationships [7, 15]. However, supportive relationships were more common than unsupportive ones in the present study, with participants emphasizing the importance of positive, normalizing interactions between parents, family, and partners. Similarly, though the impact of $\mathrm{CAH}$ on dating was noted, most participants reported being in very satisfying and supportive relationships, findings which somewhat contradict those from prior research, which found women less likely to engage in intimate relationships [7]. For both areas, the negative impact of CAH was more present in women's anxiety about disclosing or in fears of others' reactions than actual negative responses from others. 
Previously documented concerns regarding increased mental illness among individuals with $\mathrm{CAH}$ [9] were reflected in the results of this study. For those participants who saw them as CAH-related, they more often attributed them to physiological changes in mood and to external problems with the way physicians and families handled or disclosed CAH rather than from internal processes related to aspects of sexual or gender identity. It is, however, important to note that many participants saw their mental health concerns as typical and unrelated to issues of $\mathrm{CAH}$, or were unsure of a link between CAH and their emotional or mental health functioning. For example, it was unclear if one participant's substance use was a coping mechanism for negative emotions surrounding her $\mathrm{CAH}$ or if it was influenced by a family history of alcoholism or both. Similarly, though not all participants had received counseling, all shared a wide range of reasons and goals for counseling, the majority of which were unrelated to $\mathrm{CAH}$.

Unlike their experience with medical professionals, which were more negative than positive, as described both in this study and in one other [7], the reverse was true for experiences with mental health practitioners. While therapist's knowledge of CAH or willingness to learn about $\mathrm{CAH}$ was positive, our findings lent support for the greater importance of the working alliance and basic helping skills. Since issues of disclosure figured prominently in several domains, therapist empathy, openness, and listening skills cannot be emphasized enough. They become all the more important in light of findings that participants had a tendency to overlook or be reticent in telling a mental health clinician about CAH. Similarly, participants wanted both mental health and medical clinicians to be more attuned to the medical implications and the impact of $\mathrm{CAH}$ on emotions and mood regulation, which suggests that general knowledge about CAH on its own is important, but must be integrated with a tailored understanding of the individual.

A related finding, noteworthy for practitioners working with individuals with $\mathrm{CAH}$, was that six of the eight participants reported never having met anyone with CAH. As consistent with previous findings [7], this is something that contributed to feelings of isolation for the women in this study. It is unlikely that individuals would know others with CAH because the population is small in comparison to the broader population, and disclosure of this information to others is rare. Interestingly, few participants recommended that health care professionals connect them with support groups, which would seem to be one solution to deter isolation for individuals with CAH. Since many of the women in this study strove to achieve "normal" functioning and identities, the use of a support group may potentially challenge this. The domain systemic considerations included several barriers to finding individual endocrine or therapeutic care providers which may have contributed to women emphasizing this need over the need for support groups and also serves as an important link between individual relationships (e.g., between doctors and patients, or individuals and friends) and societal norms or challenges which may impact them.
7.1. Limitations. While attempts were made to examine $\mathrm{CAH}$ degree-or-type-specific factors related to HRQL, almost all of our participants were diagnosed in infancy with salt wasting type. Therefore it was not possible to highlight trends between $\mathrm{CAH}$ groups. Similarly related to sampling, participants self-selected into our study, these individuals may be relatively healthy and interested in therapeutic issues pertaining to $\mathrm{CAH}$ as compared to individuals who chose not to participate in the study. As such, our sample may not be representative of the broader $\mathrm{CAH}$ population, including those individuals who hold strong negative feelings towards $\mathrm{CAH}$ research or who do not identify strongly as a woman with CAH. Also, much of the research on CAH exists within a larger DSD literature base, and this study did not include individuals with any other DSD diagnoses. As such, we are unable to generalize beyond $\mathrm{CAH}$.

\section{Conclusions}

8.1. Recommendations for Research. Present in our findings was the importance of considering cohort effects, which participants attributed to the relative newness of $\mathrm{CAH}$ as a disease, related changes in standards of care, and unique needs of individuals dependent upon age. Indeed, future research could expand upon these findings on identity in women with $\mathrm{CAH}$ by further exploring variables related to identity development processes. That being said, given the rarity of this population, it is unlikely that recommendations from this and other qualitative studies will be meaningfully examined in a large enough sample of individuals with $\mathrm{CAH}$ using quantitative methods. Therefore, it is recommended that mixed methods or qualitative approaches employing samples of practitioners or individual counseling case studies be used in future research.

8.2. Recommendations for Medical Professionals. Perhaps the most limiting factor for women was the way their $\mathrm{CAH}$ impacted their functional well-being. Their general increased risk for illness impacted not only the activities they could engage in, but also everything from travel plans to choices about where to live. This impacted other domains of HRQL as well (e.g., socially, not wanting to be a burden on friends), yet is something that could be improved through better medical management and compliance. Physical concerns regarding medication and its side effects, especially weight gain, were often exacerbated by parents and doctors who minimized them or were hostile towards participants who brought them up. The impact of these concerns may be lessened by increased sensitivity and awareness on the part of physicians, potentially leading to greater medical compliance and, in turn, an improvement in several HRQL areas.

8.3. Recommendations for Mental Health Clinicians. Our results remind clinicians to be mindful of their assumptions and lend support to the primacy of the working alliance. Several participants noted that being forced into therapy was counterproductive towards establishing shared tasks and goals. Indeed, therapist traits and skills (e.g., empathy and appropriate disclosure) were more frequently mentioned 
than was therapist knowledge of CAH. Furthermore, it is important that clinicians not assume that $\mathrm{CAH}$ is a presenting concern for all individuals. Similarly, though some participants brought up gender by mentioning that they did indeed identify as women, only half of participants discussed components of gender identity or role. Also, only half discussed sexual orientation. Therefore, despite the gendered nature of $\mathrm{CAH}$, as well as some evidence suggesting the presence of less heterosexuality in this population [25], prior recommendations to assist clients with the examination of their gender and sexuality [7] should be tempered with an appreciation of individual clients' needs. Relatedly, clinicians should be sure to consider the diversity within this population, especially as it relates to terminology and identity (e.g., intersex, DSD, CAH, gender queer) and to recognize the importance of parallel identities (i.e., individual versus group membership identities).

Lastly, given our findings on the role of systemic challenges, we would like to further emphasize prior recommendations [7] that clinicians consider diversity issues and social stigma and serve as advocates for girls and women with CAH. From improving access to care by working around insurance limitations to challenging societal bias, there is much that professionals can do in and out of their dayto-day practice. Individuals with $\mathrm{CAH}$ are doing much to advocate for themselves as well as to help increase medicine and psychology's understanding of CAH. When ending our phone interviews, several participants shared their reasons for participating, "I want to see something really happen. I think that it's ... it's hard, you know, just growing up with it and I know that from my situation, and I want others to feel more comfortable in the future," and "I figure anything that can help with the next generation is ... you know, I'm here."

\section{Acknowledgments}

The authors would like to thank Claude Migeon, M.D., and Amy Wisniewski, Ph.D., for sharing their feedback on the study, Kristin Bertsch and Aubrey DeCarlo for their help with transcription, CARES and congenitaladrenalhyperplasia.org for graciously hosting our participant request as well as providing excellent resources and support for individuals and families affected by $\mathrm{CAH}$, Lehigh University for providing funding for the current study, and last but certainly not least, all the women who so openly shared their thoughts and feelings so that they might contribute to better care and improved quality of life for others.

\section{References}

[1] L. Sax, "How common is intersex? A response to Anne FaustoSterling," Journal of Sex Research, vol. 39, no. 3, pp. 174-178, 2002.

[2] S. Pang, M. A. Wallace, L. Hofman, et al., "Worldwide experience in newborn screening for classical congenital adrenal hyperplasia due to 21-hydroxylase deficiency," Pediatrics, vol. 81, no. 6, pp. 866-874, 1988.

[3] M. L. New, "21-hydroxylase deficiency: classical and nonclassical congenital adrenal hyperplasia," December 2009, http://www.endotext.org/.
[4] M. Hines, "Psychosexual development in individuals who have female pseudohermaphroditism," Child and Adolescent Psychiatric Clinics of North America, vol. 13, no. 3, pp. 641656, 2004.

[5] N. R. Maharaj, A. Dhai, R. Wiersma, and J. Moodley, "Intersex conditions in children and adolescents: surgical, ethical, and legal considerations," Journal of Pediatric and Adolescent Gynecology, vol. 18, no. 6, pp. 399-402, 2005.

[6] P. A. Lee, C. P. Houk, S. F. Ahmed, and I. A. Hughes, "Consensus statement on management of intersex disorders. International consensus conference on intersex," Pediatrics, vol. 118, no. 2, pp. 488-500, 2005.

[7] L. J. Guth, R. I. Witchel, S. F. Witchel, and P. A. Lee, "Relationships, sexuality, gender identity, gender roles, and self-concept of individuals who have congenital adrenal hyperplasia: a qualitative investigation," Journal of Gay and Lesbian Psychotherapy, vol. 10, no. 2, pp. 57-75, 2006.

[8] F. Youssef and R. Wong, "Educating clinicians to assess quality of life in patients with chronic illness," Home Health Care Management and Practice, vol. 15, no. 1, pp. 20-26, 2002.

[9] T. H. Johannsen, C. P. L. Ripa, E. L. Mortensen, and K. M. Main, "Quality of life in 70 women with disorders of sex development," European Journal of Endocrinology, vol. 155, no. 6, pp. 877-885, 2006.

[10] M. L. Cull, "Commentary: a support group's perspective," British Medical Journal, vol. 330, no. 7487, p. 341, 2005.

[11] G. Warne, S. Grover, J. Hutson, et al., "A long-term outcome study of intersex conditions," Journal of Pediatric Endocrinology and Metabolism, vol. 18, no. 6, pp. 555-567, 2005.

[12] U. Kuhnle, M. Bullinger, and H. P. Schwarz, "The quality of life in adult female patients with congenital adrenal hyperplasia: a comprehensive study of the impact of genital malformations and chronic disease on female patients life," European Journal of Pediatrics, vol. 154, no. 9, pp. 708-716, 1995.

[13] B. May, M. Boyle, and D. Grant, "A comparative study of sexual experiences," Journal of Health Psychology, vol. 1, no. 4, pp. 479-492, 1996.

[14] A. B. Wisniewski, C. J. Migeon, M. A. Malouf, and J. P. Gearhart, "Psychosexual outcome in women affected by congenital adrenal hyperplasia due to 21-hydroxylase deficiency," Journal of Urology, vol. 171, no. 6, pp. 2497-2501, 2004.

[15] L. M. Liao, "Learning to assist women born with atypical genitalia: journey through ignorance, taboo and dilemma," Journal of Reproductive and Infant Psychology, vol. 21, no. 3, pp. 229-238, 2003.

[16] M. E. Boyle, S. Smith, and L.-M. Liao, "Adult genital surgery for intersex: a solution to what problem?" Journal of Health Psychology, vol. 10, no. 4, pp. 573-584, 2005.

[17] U. Kuhnle and M. Bullinger, "Outcome of congenital adrenal hyperplasia," Pediatric Surgery International, vol. 12, no. 7, pp. 511-515, 1997.

[18] M. A. Malouf, C. J. Migeon, K. A. Carson, L. Petrucci, and A. B. Wisniewski, "Cognitive outcome in adult women affected by congenital adrenal hyperplasia due to 21-hydroxylase deficiency," Hormone Research, vol. 65, no. 3, pp. 142-150, 2006.

[19] C. M. Ogilvie, N. S. Crouch, G. Rumsby, S. M. Creighton, L.M. Liao, and G. S. Conway, "Congenital adrenal hyperplasia in adults: a review of medical, surgical and psychological issues," Clinical Endocrinology, vol. 64, no. 1, pp. 2-11, 2006.

[20] F. M. E. Slijper, S. L. S. Drop, J. C. Molenaar, and S. M. P. F. De Muinck Keizer-Schrama, "Long-term psychological evaluation of intersex children," Archives of Sexual Behavior, vol. 27, no. 2, pp. 125-144, 1998. 
[21] J. F. Morgan, H. Murphy, J. H. Lacey, and G. Conway, "Long term psychological outcome for women with congenital adrenal hyperplasia: cross sectional survey," British Medical Journal, vol. 330, no. 7487, pp. 340-341, 2005.

[22] C. E. Hill, B. J. Thompson, and E. N. Williams, "A guide to conducting consensual qualitative research," Counseling Psychologist, vol. 25, no. 4, pp. 517-572, 1997.

[23] C. E. Hill, B. J. Thompson, S. A. Hess, S. Knox, E. N. Williams, and N. Ladany, "Consensual qualitative research: an update," Journal of Counseling Psychology, vol. 52, no. 2, pp. 196-205, 2005.

[24] U. Kuhnle, M. Bullinger, H. P. Schwarz, and D. Knorr, "Partnership and sexuality in adult female patients with congenital adrenal hyperplasia. First results of a cross-sectional quality-of-life evaluation," Journal of Steroid Biochemistry and Molecular Biology, vol. 45, no. 1-3, pp. 123-126, 1993.

[25] H. F. L. Meyer-Bahlburg, C. Dolezal, S. W. Baker, and M. I. New, "Sexual orientation in women with classical or nonclassical congenital adrenal hyperplasia as a function of degree of prenatal androgen excess," Archives of Sexual Behavior, vol. 37, no. 1, pp. 85-99, 2008. 